AIAA 98-4339

\title{
A PILOTED SIMULATION STUDY OF WAKE TURBULENCE ON FINAL APPROACH
}

\author{
Eric C. Stewart \\ NASA Langley Research Center \\ Hampton, VA 23681
}

\begin{abstract}
A piloted simulation study has been conducted in a research simulator to provide a means to estimate the effects of different levels of wake turbulence on final approach. A worst-case methodology was used to ensure conservative estimates. Fourteen airline pilots voluntarily participated in the study and flew almost 1000 approaches. The pilots rated the subjective severity of the disturbances using a special rating scale developed for this study. Several objective measures of the airplane/pilot response to the simulated wake turbulence were also made. All the data showed a large amount of variation between pilots and to a lesser extent for a given pilot. Therefore, the data were presented at 50, 70, 90 percentile levels as a function of vortex strength. The data allow estimates of the vortex strength for a given subjective or objective response and vice versa. The results of this study appear to be more conservative than the results of previous studies.
\end{abstract}

\section{Introduction}

The National Aeronautics and Space Administration (NASA) is developing the technology for a system to safely increase airport capacity in instrument meteorological conditions (IMC). The system, called the Aircraft Vortex Spacing System or AVOSS, is designed to allow reduced airplane

*Senior Research Engineer, Member AIAA

Copyright (C) 1998 by the American Institute of Aeronautics and Astronauties, Inc. No copyright is asserted in the United States under Title 17, U.S. Code. The U.S. Government has a royalty-free license to exercise all rights under the copyright claimed herein for Government Purposes. All other rights are reserved by the copyright owner. spacing in IMC conditions in the airport terminal area. ${ }^{1}$ AVOSS will be able to predict and advise the air traffic control system when weather conditions are such that the wake will be safely transported out of the path of following airplanes. As a backup to these weather-based predictions, the system will include ground-based instrumentation to directly track the wakes and assure that all airplanes are safely separated from all wake vortex systems. Since any such instrumentation must have a wake of finite strength in order to successfully track the wake, the specifications for these instruments have to include threshold levels of vortex strength in the presence of background atmospheric turbulence. Such threshold detection levels must be low enough to guarantee safe aircraft operations in the worst-case conditions. That is, if the instrument fails to track the vortex because its strength is less than the threshold value, then that strength must be low enough that a following airplane can fly through the wake without significant effect. In addition, future vortex spacing systems may permit airplanes to deliberately fly through regions where wakes still exist. When the strength of the wake is above the tracking instrument threshold, but has decayed sufficiently, an airplane may be allowed to fly into an approach region containing the wake. A value for the acceptable level of wake strength will, therefore, be needed for these future systems.

Recognizing the need for defining values for instrument threshold and an acceptable level of wake strength, the Langley Research Center has embarked on a series of studies to more fully understand the complex interaction of an airplane with the wake of a preceding airplane. Analytical studies ${ }^{2,3,4}$, static and free-flight wind tunnel tests ${ }^{5}$, and simulated auto-land approaches ${ }^{6}$ have all studied various aspects of airplane dynamics in the presence of a wake vortex system. However, all of these studies have lacked a means or criteria to estimate the subjective effect of a given level of wake turbulence on routine airline operations. Previous piloted simulation studies ${ }^{7}$ have concentrated on defining hazardous disturbances rather than the nuisance or insignificant disturbances needed for the AVOSS system or future 
wake vortex spacing systems. These disturbances must be acceptable for routine operations for all flight crews, equipment, and ultimately the passengers.

It is this low-level wake turbulence which the present study addresses in the context of routine airline operations. The present study attempts to provide a means to estimate the vortex strength for a given subjective response or vice versa. In addition, the results of the study will make it possible to estimate the pilot's and the airplane's objective responses for the given conditions. A 10-point rating scale was developed which uses familiar atmospheric turbulence terminology. This scale was used by the pilots to evaluate various levels of wake turbulence in a piloted, motion-base simulator. In order to provide an extra margin of safety in the final estimates, a "worst-case methodology" was used in the design of the experiment. For example, the experimental scenario included poor visibility conditions, multiple wake disturbances, a short runway, and a simulated airplane with a small wing scrape angle.

A total of 14 airline pilots of various experience levels were used as test subjects. Nearly 1000 approaches were flown in the course of the study. Although the cockpit generally had a generic transport layout, the simulated aerodynamics were representative of a Boeing 737-100 airplane. A sidearm pitch and roll controller was used because a simulator with a yoke was not readily available. The principal research variable was the maximum level of the rolling acceleration produced by the vortex. Since preliminary tests (unpublished) had indicated large variations from pilot to pilot, an adaptive methodology was used to prevent either trivial or extremely difficult conditions being repeatedly presented to any test subject. Because some previous studies of wake hazards had used the ratio of vortex rolling acceleration to the maximum available roll control acceleration as a metric for determining hazardous disturbances ${ }^{8}$, roll control authority was the second primary research variable. That is, in addition to tests with the nominal Boeing 737-100 control authority, tests were also conducted with $75 \%$ and $150 \%$ of the nominal control authority. The primary data of the study were the subjective disturbance ratings taken after each approach. In addition to the subjective data, several quantitative measures of the approaches were made. Both types of data are summarized in a statistical format so that the results can be used to estimate the vortex effects at different percentile levels.

\section{Terminology}

Symbols

DR disturbance rating from rating scale in figure 4, non-dimensional

GA number of go-arounds executed, \% of total runs

$h$ altitude above ground, $\mathrm{ft}$

$\dot{h} \quad$ vertical velocity (positive upward), fps

$\mathrm{p}$ roll rate (positive to right), degrees/sec

WS number of wing scrapes experienced, $\%$ of total runs

$\mathrm{x}$ longitudinal position along runway, $\mathrm{ft}$

y lateral position relative to runway centerline (positive to right), $\mathrm{ft}$

$\delta_{l a t} \quad$ sidearm controller lateral deflection (positive right, $100 \%=20$ degrees), $\%$ of full travel

$\dot{\delta}_{\text {lat }} \quad$ sidearm controller lateral deflection rate $(100 \%=$ 50 degrees $/ \mathrm{sec}), \%$

$\Gamma_{\text {crit }}$ critical circulation strength corresponding to a rolling acceleration of $10.6 \mathrm{deg} / \mathrm{sec}^{2}, \mathrm{ft}^{2} / \mathrm{sec}$

$\Gamma_{0} \quad$ initial circulation strength before decay, $\mathrm{ft}^{2} / \mathrm{sec}$

$\phi \quad$ roll angle (positive right wing down), degrees

$\ddot{\phi}_{v} \quad$ roll acceleration due to vortex (positive right wing down), degrees $/ \mathrm{sec}^{2}$

$|\xi| \quad$ absolute drift angle $=\mid($ ground track angle $)$ (heading)|, degrees

Subscripts

err error

f follower airplane

g generator airplane

td touchdown

$\mathrm{v}$ vortex

Abbreviations

abs absolute value

AVOSS aircraft vortex spacing system

CRT cathode ray tube

IMC instrument meteorological conditions

Max maximum

RMS root mean squared value

VMC visual meteorological conditions

VMS visual motion simulator

\section{Simulator}

Hardware: The study was conducted in the Langley Visual Motion Simulator (VMS). The VMS is a general-purpose simulator consisting of a two-person cockpit mounted on a six-degree-of-freedom synergistic motion base, Figure 1. The equations of motion were solved on a HP/Convex C3840 Computer running with 


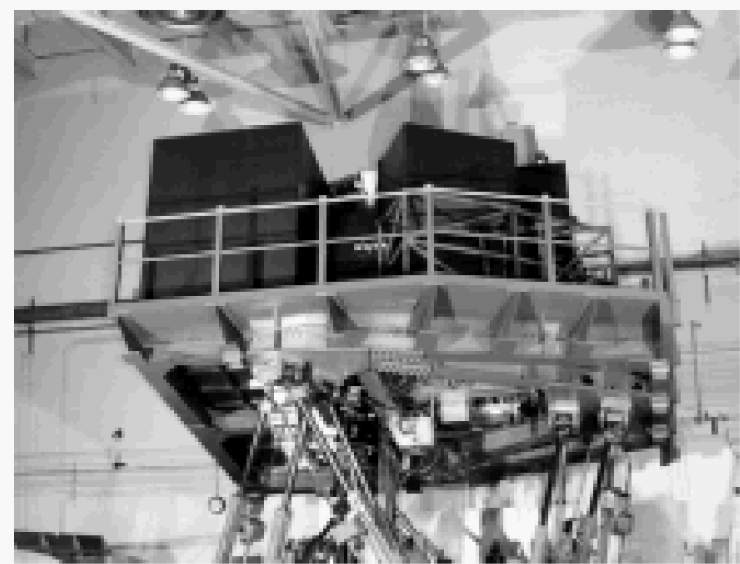

Figure 1. Exterior view VMS simulator

an iteration rate of 32 cycles per second. Motion cues were provided in the simulator by computer algorithms that commanded the relative extension or retraction of the six legs (hydraulic actuators) of the motion base.

The cockpit, Figure 2, had four collimatedimage visual systems, a center console with four throttles (only two were active), a hydraulically loaded 2-axis McFadden sidearm controller, hydraulically-loaded rudder pedals, an electronic flight instrument panel, as well as a sound system. Two of the four visual systems provided views through the forward windows,

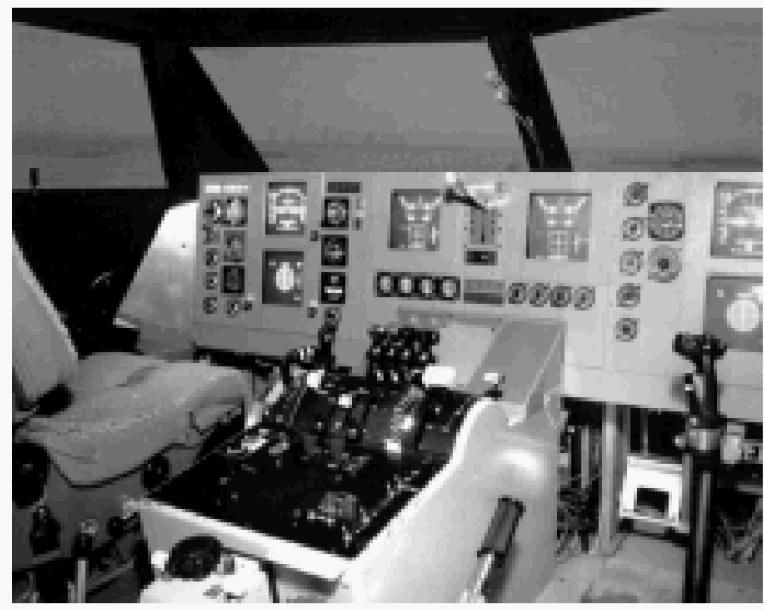

Figure 2. View of VMS cockpit.

and the other two provided views through the side windows. The test subject in the left seat could only see the scenes out of the forward-looking system and the left-looking system on the left side of the cockpit. Each of the four visual systems had a field of view of approximately 40 degrees horizontally by 22 degrees vertically. All four visual out-the-window scenes were generated by a 5 -channel Evans and Sutherland ESIG 3000/GT Advanced Computer Generated Image system.
The main instrument panel included six color graphics electronic CRT displays using a Calligraphic Raster Display System driven by an Eagle 1000 from Terabit Computer Engineering with an iteration rate of 48 cycles per seconds. The primary flight display, Figure 3, included an artificial horizon, airspeed and

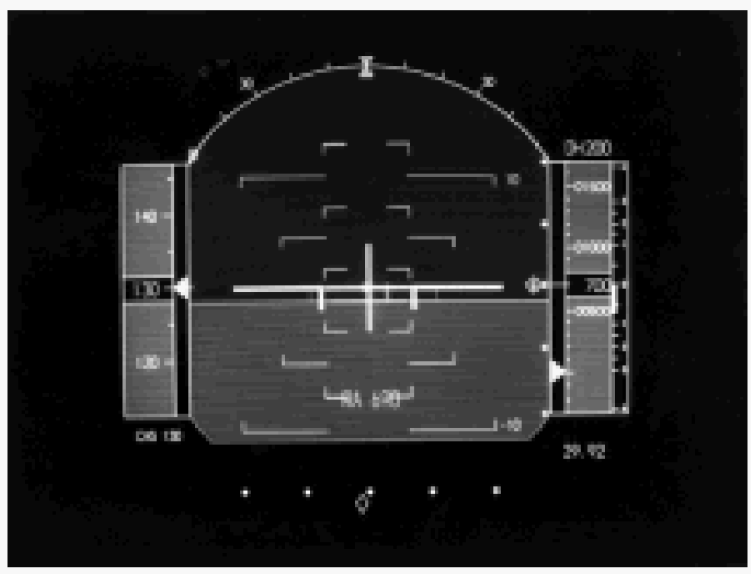

Figure 3. Primary flight display.

altitude tapes, localizer and glide-slope indicators, as well as a dual command flight director.

The two-axis (pitch and roll) McFadden sidearm controller was used because a simulator with a yoke was not readily available. The controller had a physical travel limit of $+/-20$ degrees and a linear force gradient. At the maximum travel of 20 degrees, the roll control forces was $8.7 \mathrm{lbs}$. measured at moment arm of 6 inches from the pivot point. A special analog rate limiting circuit was used to limit the maximum control rate to 50 degrees $/ \mathrm{sec}$ in the roll axis. This circuit was added to assure that the pilot could not move the cockpit controller faster than the simulated B737 control system could move the simulated aerodynamic surfaces.

The transport lags of the visual scene and the primary flight display were estimated to be approximately $100 \mathrm{~ms}^{9}{ }^{9}$ The motion base had a phase lag of approximately 11 degrees throughout the range of 0 to 2 Hz. ${ }^{10}$

Software: The airplane math model used was representative of a Boeing 737-100. Although the cockpit was equipped with a sidearm controller, the simulated control system was similar to that of a 737-100 equipped with a yoke. That is, there was no control augmentation other than a yaw damper and an auto-throttle system. A generic algorithm that did not duplicate any known commercial equipment drove the flight director. In addition to simulating these fundamental airplane characteristics, the main computer also served to record the quantitative research data such as time histories of key 
airplane states as well as the conditions at events such as touchdown, wing scrape, and go-around.

\section{Test Subjects}

The fourteen test subjects were all active airline pilots who flew both domestic and international routes. Although they voluntarily participated in these tests, they were paid a nominal stipend for their services plus travel and living expenses. They held a variety of positions, operated different types of equipment, and had different routes. Although they voluntarily participated in these tests, they were paid a nominal stipend for their services plus travel and living expenses. They held a variety of positions, operated different types of equipment, and had different levels of total experience, Table I. All test subjects were males.

Table I. Pilots used in Study

\begin{tabular}{|c|c|c|c|}
\hline Pilot & Total hours & Position & Equipment \\
\hline 1 & 12,000 & C & $737-300 / 400$ \\
\hline 2 & 7,500 & FO & $757 / 767$ \\
\hline 3 & 10,000 & $\mathrm{FO}$ & 767 \\
\hline 4 & 6,500 & $\mathrm{FO}$ & $757 / 767$ \\
\hline 5 & 8,400 & $\mathrm{C}$ & DC- $10-30 / 10$ \\
\hline 6 & 3,800 & $\mathrm{FO}$ & DC-9-82/83 \\
\hline 7 & 14,000 & $\mathrm{FO}$ & $767-300$ \\
\hline 8 & 12,000 & FO & $737-400$ \\
\hline 9 & 11,700 & $\mathrm{C}$ & $737-300 / 400$ \\
\hline 10 & 9,000 & $\mathrm{C} / \mathrm{CA}$ & F100 \\
\hline 11 & 12,000 & $\mathrm{C} / \mathrm{l}$ & 757 \\
\hline 12 & 3,700 & $\mathrm{FO}$ & 747 \\
\hline 13 & 5,000 & 1 & A320 \\
\hline 14 & 10,000 & $\mathrm{FO}$ & 767 \\
\hline
\end{tabular}

\section{Rating Scale}

A special rating scale, figure 4 , was developed for these tests. The scale was developed in consultation with several members of the AVOSS team including highly experienced pilots intimately familiar with the test program. Since it was intended to be used by professional airline pilots, it used familiar atmospheric turbulence terminology and analogies. It was decided to eliminate all references to "acceptable" disturbances since such judgments were deemed to be highly dependent on the different perspectives of the test subjects.

\section{Tests}

Worst -Case Methodology: In order to provide an extra margin of safety in the results, a worst-case approach was used in the selection of four of the experimental conditions.

First, rather than the typical single disturbance of past studies, a more difficult scenario consisting of repeated disturbances was used. These disturbances simulated a wake that was randomly "snaking" back and forth across the -3 degree Instrument Landing System (ILS) approach corridor. Thus, the pilot had to cope with multiple disturbances with randomly varying direction, onset rates, and reversals. The pattern of the disturbance was determined by random number generators. A set of 7 disturbance patterns that were judged to be particularly difficult in preliminary tests, ${ }^{11}$ was used for all data maneuvers. These seven disturbance patterns were randomly mixed with six different vortex strength levels which were also randomly distributed from run to run. The resulting mix of disturbance patterns and vortex strength levels were intended to prevent the pilots from anticipating the disturbances. The second aspect of the worst-case methodology was to use a manually flown final approach in Instrument Meteorological Conditions (IMC). Thirdly, a simulated runway of only $7000 \mathrm{ft}$ length was used. This prevented the pilots from correcting large lateral position offsets by landing past the nominal touchdown point on a long runway. The fourth and final element of the worst-case methodology was in the selection of a simulated wing scrape angle. A conservative wing scrape angle of 8 degrees was used in all tests. This value is less than most, but not all, transport airplanes.

Pilot Training: Approximately one week before the test subjects were to arrive for their simulation session, they received a letter explaining the purpose of the tests and an approximate schedule of the day's activities. A copy of the rating scale (Figure 4) was also included with an explanation of how the rating scale was to be used. Each test subject was able to complete his session in one 10- to 11-hour working day including several break periods. When a test subject arrived at the simulator for their session in the morning, he was given a one-hour briefing which explained the AVOSS program and the purpose and procedures of the tests.

The simulation environment contrasted sharply with the present-day operational environment in which the pilot has very limited knowledge of the maximum strength of the wake in his approach corridor or how near the area of maximum strength he may have approached. With this limited knowledge of wake turbulence, good judgment often dictates that a go- around be executed at the 
1. GLASSY $(2 \%$ smaller*): Flight conditions are glassy smooth. There is no detectable turbulence and winds are completely still to very light. It is a still, clear morning when the airplane seems to fly itself.

2. SMOOTH ( $10 \%$ smaller $\left.^{*}\right)$ : There may be an almost imperceptible disturbance and winds are light and steady. Only slight control inputs are required to maintain flight path. Conditions are representative of a nighttime stable atmosphere.

3. CALM (20\% smaller $\left.{ }^{\star}\right)$ : Pilot may be aware of very small disturbances, but effects require little if any compensation. Conditions are typical of fair weather flying.

4. PERCEPTIBLE (40\% smaller $\left.{ }^{*}\right)$ : Small pilot control inputs may be required to compensate for disturbances. Thermal activity has become recognizable.

5. SLIGHT (70\% smaller*): Conscious pilot control inputs are occasionally required to compensate for disturbances. Conditions are only slightly less favorable than average weather conditions, but it is still a "good day to fly."

6. SMALL (95\% smaller*): Definite pilot control inputs are required for most of the maneuver to compensate for disturbances. Conditions are typical of mid afternoon summer-time conditions.

7. MODERATE $\left(99 \%\right.$ smaller $\left.^{\star}\right)$ : Moderate control inputs are frequently required to maintain flight path and attitude. Crew and passengers are aware of conditions. Conditions are representative of approaches conducted in the vicinity of frontal activity.

8. LARGE (99.5\% smaller*): Large control inputs are continually required to maintain flight path and attitude. Some passengers may become ill. Conditions are unusual, even for approaches conducted in the vicinity of frontal activity.

9. SAFE LIMIT (99.9\% smaller*): Aggressive control inputs are required to maintain flight path. Crew is alert for wind shear conditions. Some passengers will probably become ill and some will complain about the approach. Flight Attendants will discuss conditions after landing. Conditions are representative of approaches conducted in the vicinity of thunderstorms.

10. UNSAFE (99.99\% smaller*): Airplane response to control input is insufficient to contain effects of disturbance. Good judgment dictates a missed approach. If the approach is continued, the subsequent landing may be hard, or displaced from the centerline of the runway or from the touchdown zone. Airplane damage may be incurred.

*Numbers are estimated percentages of normal flight operations with smaller disturbances.

Figure 4. Disturbance Rating Scale used to evaluate wake vortex disturbances.

first sign of wake turbulence. However, in this simulation study the pilots were instructed to assume they were operating in a terminal area equipped with an AVOSS-like system. This system had measured the strength of the wake turbulence and determined it was not severely hazardous for his simulated airplane. Further, the pilot was told that his airplane would fly through the area of maximum strength during the first disturbance experienced in a given run. Later disturbances in the run would not be drastically different as they might be in today's operational environment. Therefore, the pilots were instructed to fly the approaches as long as they thought they could make a good landing from their present state assuming they would have no more disturbances. That is, they were not to initiate an immediate go-around after a disturbance at a high altitude because they thought a similar or larger disturbance would be unsatisfactory close to the ground.

After the briefing, the tests subjects were taken to the simulator where they flew a series of approaches to get them acclimated to the simulator and the test procedures. These practice approaches lasted about one hour. They started with visual conditions and mild wind conditions and progressed to instrument visual conditions, random atmospheric turbulence, and slight wind shears. Finally, a short series of simulated vortex disturbances was conducted immediately before the research runs.

Approach Conditions: All the research (datarecording) runs were initiated approximately $2 \mathrm{NM}$ from the threshold of the 7000-ft runway at an altitude above the ground of $700 \mathrm{ft}$. Although the airplane was exactly stabilized on a -3 degree flight path, a $50 \mathrm{ft}$ lateral offset was introduced to give the pilot a small nominal task. The auto-throttles and yaw damper were engaged. The flaps and landing gear were extended in the final landing configuration, and the indicated airspeed was 130 knots or 1.3 times the stall speed. The simulated visual conditions were representative of Instrument Meteorological Conditions (IMC) with a gradual transition to Visual Meteorological Conditions (VMC) around an altitude of $200 \mathrm{ft}$ above the ground. A light wind ( 5 knots at 45 degrees from the runway 
heading) was simulated along with random almost imperceptible atmospheric turbulence.

The encounter geometry simulated the condition where the airplane's flight path was nearly aligned with the rotation axis of the vortex such that the primary disturbance occurred in the airplane's roll axis. To prevent the pilot from anticipating the airplane's response and to make the task more difficult, the airplane was subjected to repeated, random disturbances in any given run as if the wake were meandering back and forth across the -3 degree flight path, Figure 5 . Thus, the pilot had to continually

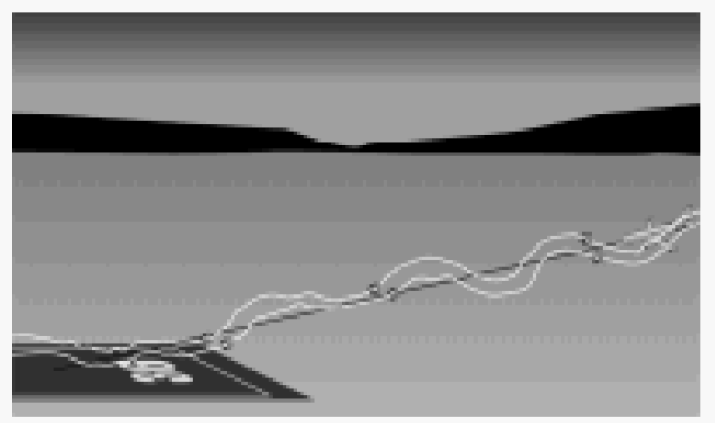

Figure 5. Worst-case encounter geometry.

contend with random roll upsets in both directions with varying onset rates and durations.

From the pilot's perspective each approach naturally divided itself into three different segments called the IMC, VMC, and Landing segments. The IMC segment was flown entirely on instruments with the flight director the primary area of focus. The VMC segment was also called the transition segment because the pilot typically was shifting his point of attention back and forth from the flight director to the visual presentation of the runway out the front window as visibility gradually started to improve at an altitude of around 200 feet. The VMC segment ended and the Landing segment began when the numbers on the end of the runway passed out of view at an altitude of about 50 feet. The Landing segment included the flare and touchdown. The run was terminated approximately 5 seconds after touchdown. After the run was terminated, the pilot used the rating scale to rate each of the three segments of the just-completed approach. He was free to rate each segment independently.

Research Variables: The primary research variable was the normalized vortex strength (or simply "normalized strength" or "vortex strength"). This nondimensional ratio can be interpreted in at least two different ways. First, it is simply the ratio of the maximum rolling acceleration produced by the vortex to the rolling acceleration produced by full deflection of the lateral controls (with the nominal lateral control authority as explained below). Alternately, the normalized strength can be interpreted as the ratio of the steady-state rolling velocity produced by the vortex to the steady-state rolling velocity produced by full deflection of the lateral controls. This alternate interpretation is based on the classical one-degree-offreedom approximation to the rolling mode response of an airplane. Full deflection of the lateral controller of the simulated Boeing 737-100 with the nominal lateral control authority produced an initial acceleration of 53 degrees $/ \mathrm{sec}^{2}$ and a steady-state velocity of 32 degrees/second. Thus, a normalized

Table II. Equivalent Expressions of Vortex Strength

\begin{tabular}{|c|c|c|}
\hline $\begin{array}{c}\text { Normalized } \\
\text { Vortex } \\
\text { Strength }\end{array}$ & $\begin{array}{c}\text { Rolling } \\
\text { Acceleration, } \\
\ddot{\phi_{v}}\end{array}$ & $\begin{array}{c}\text { Rolling } \\
\text { Velocity, } \\
\mathrm{p}_{\mathrm{v}}\end{array}$ \\
\hline 1.0 & $53 \mathrm{deg} / \mathrm{sec}^{2}$ & $32 \mathrm{deg} / \mathrm{sec}$ \\
\hline
\end{tabular}

vortex strength of 1.0 is equivalent to these values as shown in Table II. The results in this paper are presented in terms of the normalized vortex strength, but they can be translated into an equivalent acceleration or velocity by multiplying the normalized strength times the acceleration and velocity values given in the table. It should be emphasized that the normalized strength is defined in terms of the maximum rolling acceleration produced by the vortex and the acceleration produced by full deflection of the lateral controls with the nominal control authority. The vortex acceleration used in the normalized vortex strength was not an average or RMS value. As explained earlier, the instantaneous values of the rolling acceleration produced by the vortex were described by a random pattern. However, the maximum or peak value of the rolling acceleration was constant during any given run and was achieved only a fraction of the time in each run. The use of the maximum or peak value is consistent with the requirements of a wake spacing system such as AVOSS because any such system will only be able to predict or measure the maximum normalized vortex strength. No system will be able to predict or measure in real-time the instantaneous acceleration because it is dependent on the instantaneous position and attitude of the encountering airplane relative to the wake.

The second research variable was the lateral control authority. In addition to the nominal lateral control, values of $75 \%$ and $150 \%$ of the nominal value 
were also tested. (The reader should keep in mind that all values for normalized strength reported herein are based on the nominal lateral control authority) This second research variable was tested to determine if the pilot would tolerate larger disturbances if he had more control authority and vice versa. In order to ensure that the order of presentation of the three levels of lateral control authority $(75 \%, 100 \%$, and $150 \%)$ did not affect the results, the order of presentation was varied from pilot to pilot. Since there are 6 permutations possible ( 3 control levels taken 3 at a time), a minimum of 12 pilots was used to provide one replication for each possible permutation.

Test Procedure: Usually each pilot was given 24 approaches at each of the three levels of lateral control authority. (The 24 approaches consisted of four replications of six disturbance levels) In the beginning of the tests some pilots were given fewer than 24 runs when their disturbance ratings were consistent for several values of normalized strength. For example, the first two pilots were used to refine the experimental procedures and were given fewer runs. The last 12 pilots were initially considered to be the primary test subjects although later it was decided to include the data from all 14 pilots.

An adaptive test procedure was used to accommodate the wide variation in pilot tolerance levels of the normalized strength. Each pilot was first given a nominal set of six different levels of normalized strength ranging from 0 to .33 for his first six approaches. His disturbance ratings were recorded after each approach. After the $6^{\text {th }}$ approach a simple spreadsheet program calculated a least squares estimate of a straight line fit to the normalized strengths and their corresponding average ratings (average of the IMC, VMC, and Landing ratings for each approach). The estimated intercept and slope of the straight line were then used to determine the next test level of normalized strength. That is, the linear equation was solved for the next strength level using the estimated coefficients and an assumed value of the disturbance rating from a random sequence from 3 to 8 . After each subsequent run the coefficients were updated, and a new level of normalized strength was determined for the next run. By using this procedure a pilot who initially rated all the disturbances on the low end of the scale (e.g. 3, 4, and 5 ), would be given larger disturbances on subsequent runs to determine the limit of his tolerance to larger disturbances. Likewise, if the pilot initially rated all the disturbances on the high end of the scale (e.g. 6, 7, 8, or higher), then he would be given smaller disturbances on subsequent runs.

After all the day's simulation runs were completed, a short debriefing was conducted to discuss the day's activities. During this debriefing the pilots were asked to estimate what numerical disturbance rating on the rating scale corresponded to a disturbance which they would personally consider as (1) insignificant and (2) acceptable for everyday routine operations.

\section{Analysis of Data}

The first step was to perform an analysis of variance on the pilot rating data to determine if there were significant differences due to the three levels of lateral control power or the three different maneuver segments. When this analysis showed no significant differences, all the rating data were put in one file for all 14 pilots and listed by the normalized vortex strength. These data were then sorted according to ascending normalized vortex strength and separated into bins with a width of 0.05 starting with the 0.00 to 0.05 bin. The data in each of these normalized strength bins were then sorted again to determine the 50, 70, and 90 percentile values of the pilot ratings. Finally, the three percentile values of the ratings were plotted at the midpoint of each normalized strength range.

The quantitative data for each approach were summarized by computing the RMS values and the maximum absolute values. These data were then sorted and plotted in the same manner as that used for the rating data.

The touchdown condition data were also analyzed in similar fashion. However, the data had to be first adjusted for the fact that a number of approaches resulted in a go-around. For example, if a go-around was executed the roll angle at touchdown was assumed to be very large.

The rates at which go-arounds and wingscrapes occurred were also calculated for each midpoint of the normalized strength bins.

\section{Results and Discussion}

A typical approach is shown in figure 6 


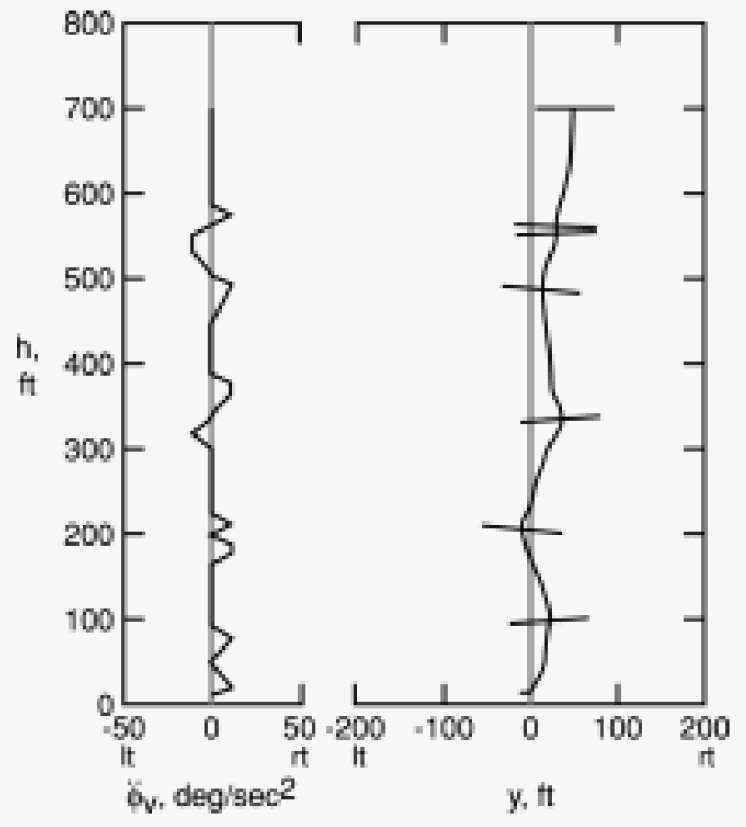

Figure 6. Typical disturbance and trajectory.

The right side of the figure shows the simulated trajectory as it might appear to an observer positioned on the far centerline of the approach end of the runway and watching the approach from behind. Superimposed on the trajectory are near-horizontal lines drawn to represent (the roll angle of) the airplane's wings. The positions of wings that were drawn were picked to show the largest roll angles that occurred in the run. The left side of the figure shows the rolling accelerations that simulated the repeated vortex disturbances. The random wave shape of the disturbance is clearly illustrated. The run started at an altitude of $700 \mathrm{ft}$ with the wings level and at a $50 \mathrm{ft}$ offset to the right of the centerline as stated earlier. The first disturbance occurs at approximately an altitude of 600 feet and rolls the airplane to the right. The effects of the disturbances can be analyzed throughout the approach. For example, at an altitude of about $400 \mathrm{ft}$ the airplane experiences a rolling acceleration to the right that momentarily displaces the trajectory to the right. The pilot countered this roll to the right with an even larger roll to the left at an altitude of about $330 \mathrm{ft}$ possibly illustrating the common problem of over controlling. It is also apparent that the pilot was correcting for lateral translation drifts as he transitioned from the flight director to out-the-window visual cues below an altitude of $200 \mathrm{ft}$. He first drifts left at $200 \mathrm{ft}$; then he drifts right until he makes a large left roll input at about 100 $\mathrm{ft}$; and it appears that he touched down on the runway with a slight translation rate to the left.
Disturbance Ratings: The analysis of variance test failed to shown any significant difference in the ratings due to either the three lateral control power levels or the three different approach segments. There were obvious differences in the ratings due to different pilots or due to the different vortex strength levels, but the other two factors made no significant difference at the 90 percent confidence level.

This lack of consistent trends in the quantitative rating data was compatible with the pilots' qualitative comments. Some pilots preferred the highest control authority while others preferred one of the other control authorities. The higher control authorities made it easy to control large disturbances, but they also were conducive to over-control problems. Although most of the pilots indicated the landing segment of the maneuver was the most difficult to execute, other pilots indicated that the wealth of natural visual cues in the landing made it easier to control the airplane.

Therefore, some pilots consistently rated the landing segment better (lower rating numbers) than the other two segments while for other pilots the opposite was true.

The disturbance ratings given by two pilots for the IMC segment with the nominal lateral control authority are presented in figure 7. Pilot No. 3 consistently gave lower ratings at all

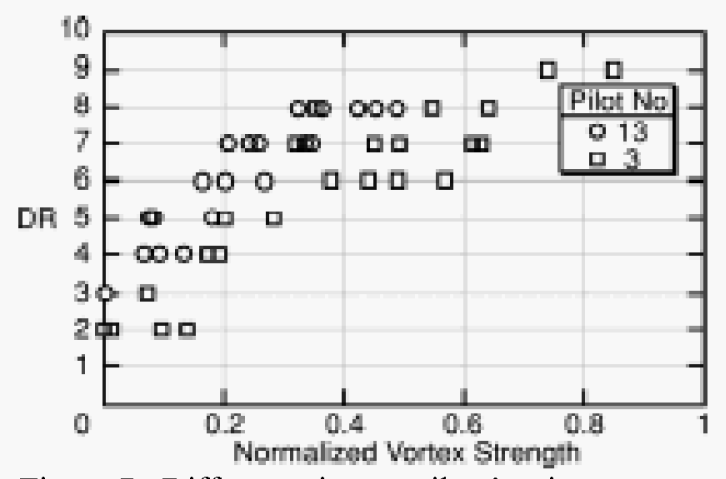

Figure 7. Difference in two pilots' ratings.

vortex strength levels. Thus, Pilot No. 3 was exposed to higher strength levels than Pilot No. 13 due to the adaptive test procedure described earlier. It also appears that Pilot No. 3 was not as consistent as Pilot No.13. That is, there is a lot more scatter in Pilot No. 3's ratings than in Pilot No. 13's ratings.

This scatter is even more apparent when all 3000 ratings ( 3 ratings for each approach) given by all 14 test subjects are shown in one figure, figure 8 . For example, at a strength of 2 (equivalent to $\ddot{\phi}_{v}=10.6$ degrees $/ \mathrm{sec}^{2}$ or $p_{v}=6.5$ degrees $/ \mathrm{sec}$ ) the disturbance ratings varied from a low of 2 to a high of 10 . This scatter is due to several factors including (1) variations 


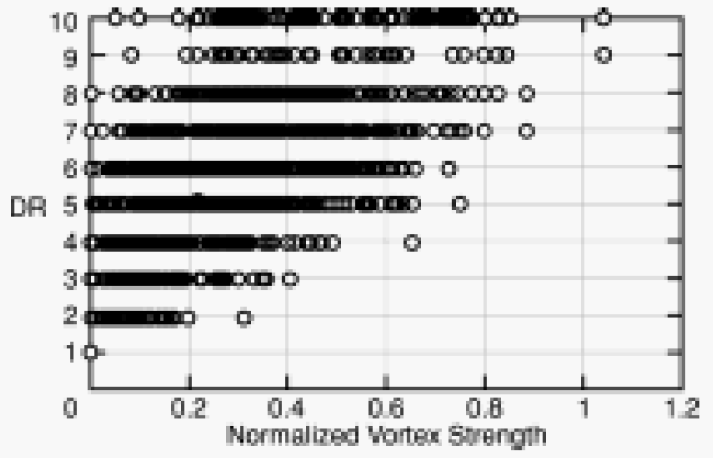

Figure 8. All disturbance ratings.

between pilots, (2) inconsistency of a given pilot for identical conditions, and (3) the differences in the random wave shapes for a given strength. The first two factors are to be expected and must be accounted for in establishing any criteria for wake disturbances. The third factor can be thought of as the cost of preventing the pilots from developing unrealistic lead in their response to a disturbance. If the pilots had encountered the same wave shape on every run, they would have quickly learned how to anticipate the response and thus been able to maintain unrealistic good control of the airplane. It was thought that it was more important to prevent the pilots from having unrealistic "learned" responses than it was to have slightly different disturbance scenarios and sacrifice some repeatability. Of course, in any systematic study of wake disturbances such as this one conducted at a research center, the element of surprise is impossible to simulate since all the test subjects know beforehand the subject of the study. This deficiency makes it even more crucial to have random scenarios that the pilots cannot anticipate.

A better approach would be to impose a small number of unannounced wake disturbances on a large number of test subjects and a wide range of equipment. The only practical way to conduct such a study would be to include the wake disturbances as part of the pilot's regular annual simulator training sessions. In this way typical wake disturbances could be used, and the pilots would be operating equipment they were completely familiar with. Such a study would require the active involvement of at least one major airline company.

It should be realized when examining the data in figure 8, that many of the data symbols are plotted on top of other symbols. The bulk of the ratings are in the center of the plot so this presentation tends to emphasize the extreme points. On the other hand, such rare extreme points are often of interest in safety related investigations.

Three percentile levels of the 3000 disturbance ratings are shown in figure 9 . The reader should be aware that the horizontal scale extends only to a value of 0.5 while that of the previous figure extended to a value of 1.2. In addition, the lines on the plots were manually faired through the data to improve the visual appearance. The data show that vortex strengths over 0.4 or 0.5 are out of the range of interest for a significant number of pilots since the 90 percentile rating at strength of 0.375 is 10 ("unsafe"). The data in

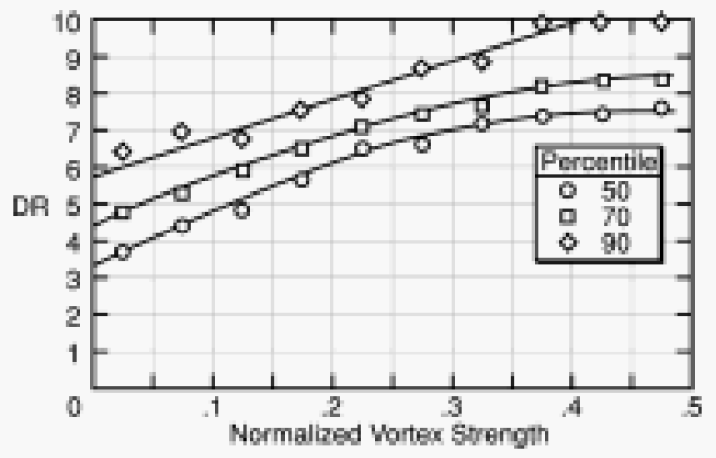

Figure 9. Summary of disturbance ratings

figure 9 along with the rating scale adjectives in figure 4 provide a means to estimate the subjective effect of a given vortex disturbance at three percentile levels.

It is significant that at a vortex strength of zero, the 90-percentile rating was approximately equal to " 6 ", and the primary adjective for a " 6 " is "small." In fact, as can be seen in figure 8 there was one rating of " 8 " at a normalized vortex strength of zero. Some pilots

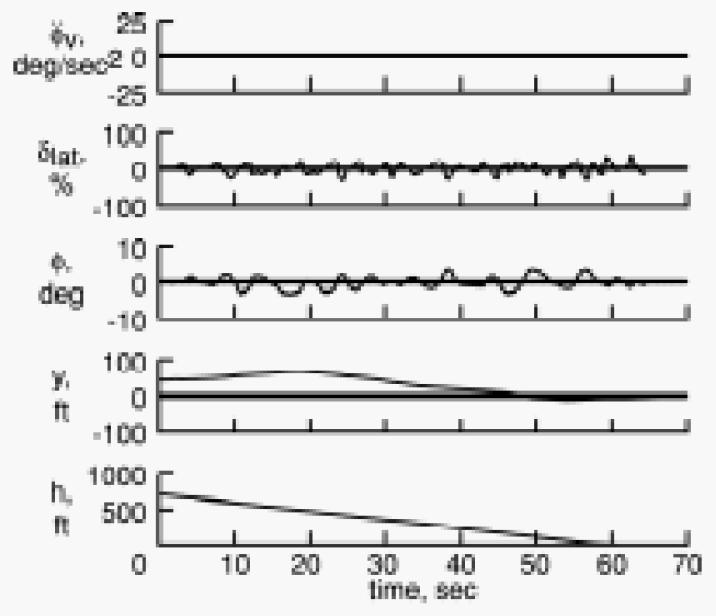

Figure 10. Approach with zero wake turbulence.

were under the impression that they were encountering "small" vortices when actually there was no disturbance. For example, Pilot No. 9 rated the approach corresponding to figure 10 as a " 6 ." As can 
be seen in the figure, the pilot was making continuous low-amplitude lateral control inputs similar to a pilot induced oscillation. When questioned about this rating during the debriefing at the end of the day, the pilot could offer no explanation as to why he had given such high ratings. However, other pilots readily recognized that there was no vortex disturbance under similar conditions and rated the same conditions as a " 2 " or "3." These low ratings were what had been initially expected since the simulated crosswind and atmospheric turbulence were negligible. Although the simulator had acceptable transport lags in the order of $100 \mathrm{~ms},{ }^{9}$ the flight director algorithm had a first-order filter with a 2second time constant. This time constant and the sidearm controller may have helped contribute to the pilot induced roll oscillations noted above. It, therefore, appears that the high disturbance ratings at zero vortex strength may be due mostly to simulator characteristics, the sidearm controller, pilot technique, pilot inexperience in using a subjective rating scale, and the general predisposition of some pilots to give high ratings when wake turbulence was a possibility.

Performance data: The quantitative performance data are summarized in figures 11 through 14. As expected all performance

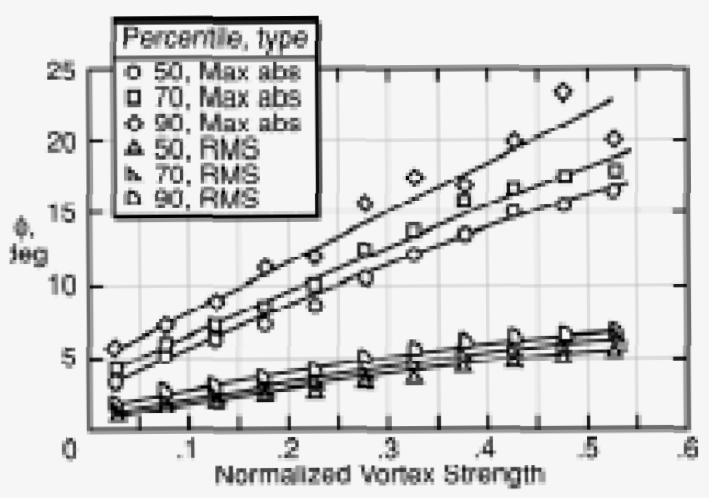

Figure 11. Roll attitude.

parameters increase with increasing vortex strength. The control position and rate data, figures 13 and 14, show that the pilots were frequently hitting the limits, especially the rate limit. The sidearm controller made it easy to go from one stop to the other because the total physical travel was relatively small, being only about 3.8 inches from stop to stop at the center of the controller handle at a radius of 6 inches from the pivot point. The special rate limiting circuit

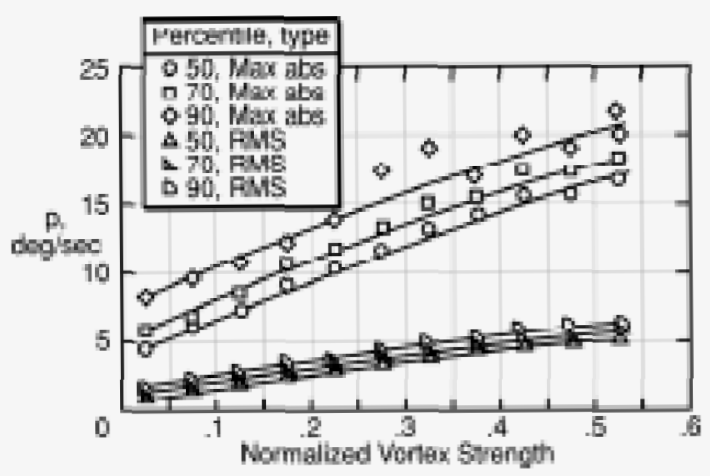

Figure 12. Rolling rate.

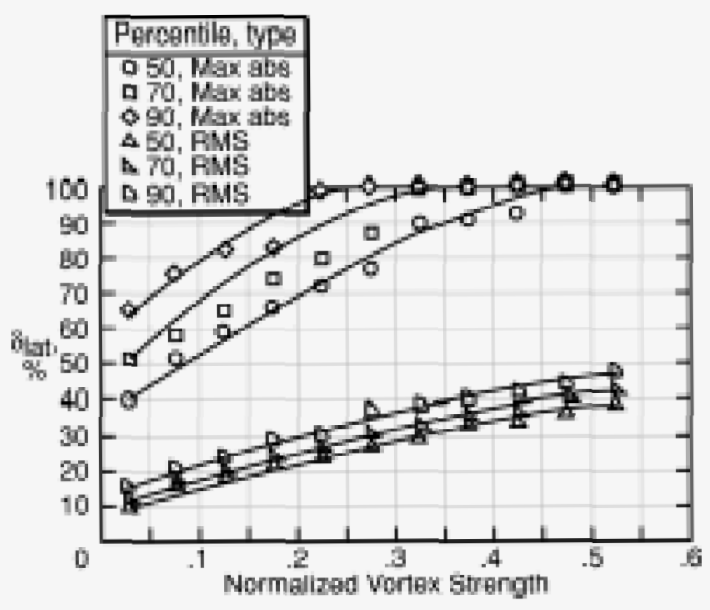

Figure 13. Lateral controller displacement.

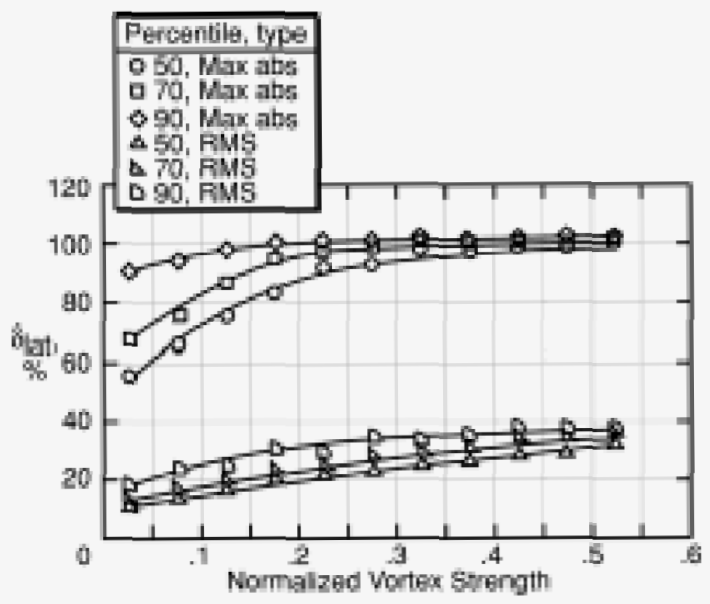

Figure 14. Lateral controller rate.

was a factor at the 90 percentile level for a relatively low vortex strength of only 0.1 . The pilots evidently desired very quick corrections to offset the vortex disturbances. Such quick corrections may have led to some of the over-controlling problems experienced by some of the pilots. The sidearm controller may have 
made it easier to control some disturbances than it would have been with a large yoke controller, but that advantage may have been offset by over-controlling problems. Additional tests with a conventional yoke controller are thus desirable.

The quantitative touchdown data are summarized in figures 15 through 19 . The

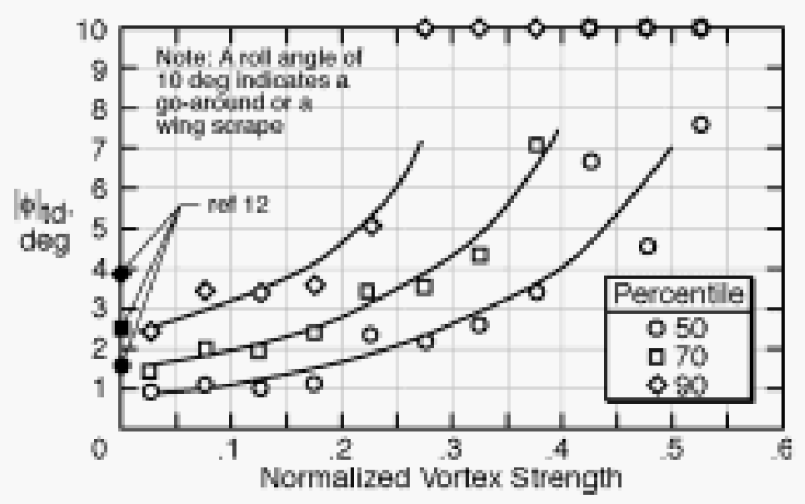

Figure 15. Roll attitude at touchdown.

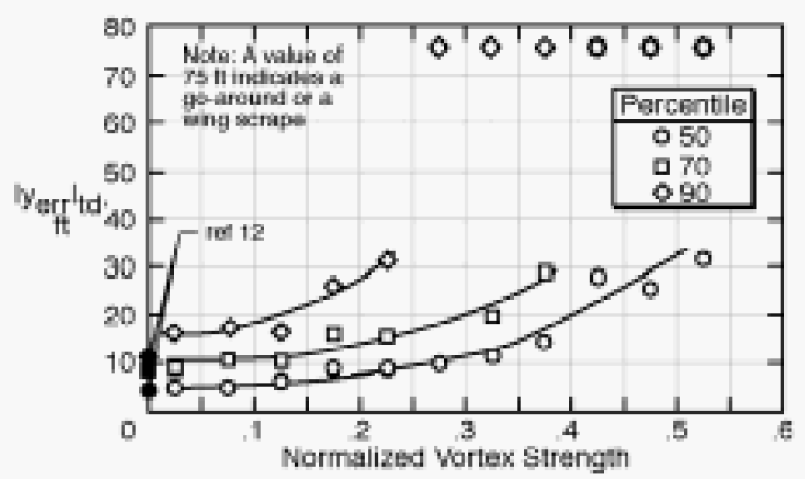

Figure 16. Lateral position error at touchdown.

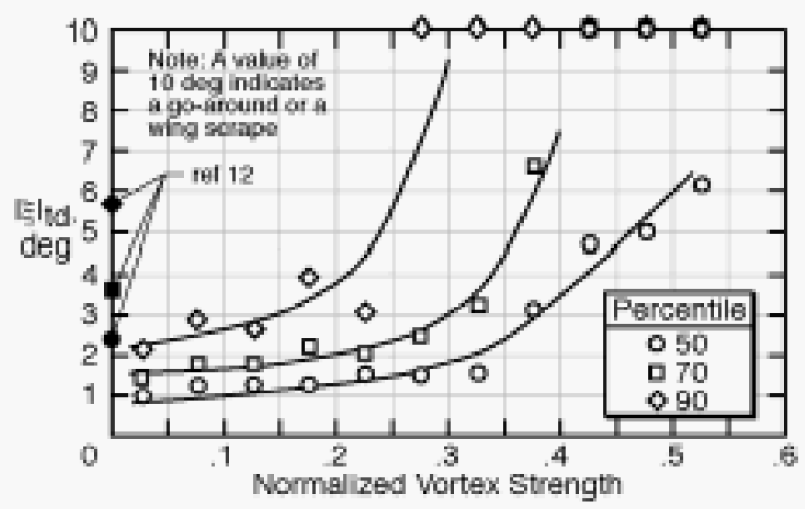

Figure 17. Drift angle at touchdown.

touchdown parameters appear to be relatively unaffected by the vortex strength up to a value of about
0.2. Above 0.2 , however, the slopes of the curves rapidly increase. This may indicate that

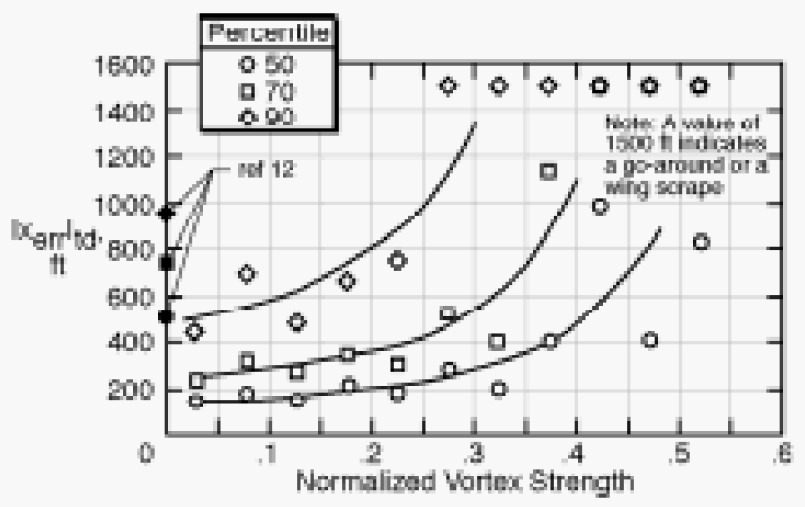

Figure 18. Longitudinal position error at touchdown.

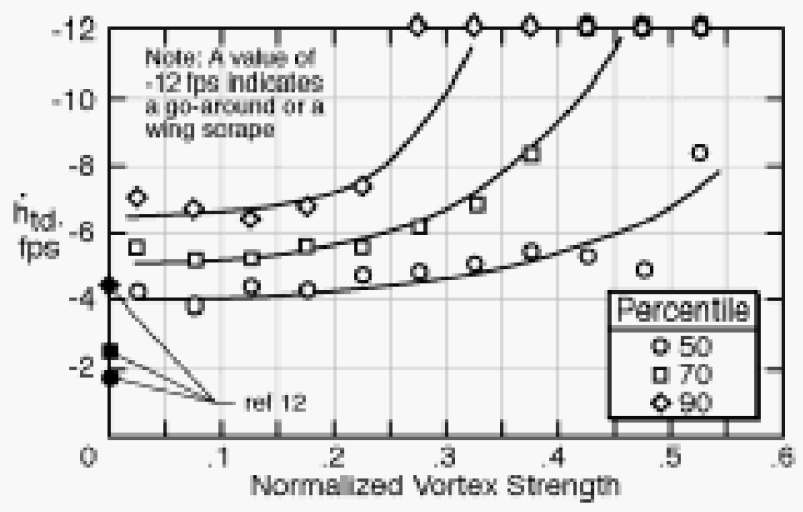

Figure 19. Sink rate at touchdown.

some of the pilots were losing their capability to control the airplane as well as they desired at the higher levels of vortex strength.

Operational touchdown data for narrow body transports ${ }^{12}$ are included on figures 15 through 19 at zero vortex strength. Comparison of these data to the simulation data extrapolated to zero vortex strengths indicates some interesting differences. For example, the roll attitude in the simulation was controlled better possibly due to the pilots' sensitivity to the low simulation scrape angle. The lateral error in the simulation was higher possibly also because of the low scrape angle which forced the pilots to forgo corrections near the ground. However, the drift angle in simulation was much better than for the operational situation. The $\mathrm{x}$ touchdown dispersions were also better in the simulator, but this was probably due to the $7,000-\mathrm{ft}$ runway used in these studies compared to the $10,000-\mathrm{ft}$ runway used for the operational data. Finally, the vertical velocities in the simulation were much higher, probably because of the lack of visual depth cues in the simulator. On balance, it appears that the simulation 
touchdown results are reasonable and the trends with increasing vortex strength are believable.

The rates at which go-arounds and wingscrapes occurred are shown in figures 20 and 21, respectively. Except for very rare cases,

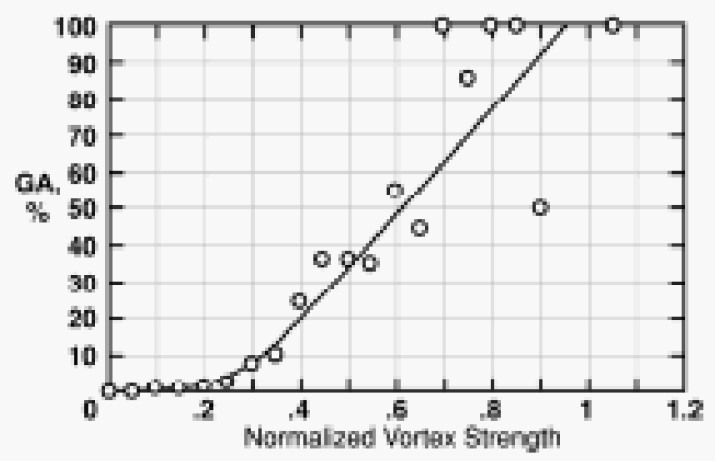

Figure 20. Rate of go-arounds.

there were no go-arounds below a normalized vortex strength of 0.2 . The explanation for these cases is probably the previously mentioned tendency for pilot induced oscillations at low or zero vortex strengths. At a vortex strength of 0.8 , almost $100 \%$ of the approaches flown resulted in a go-around. The primary reason for executing a go-around was a lateral position offset which was too large to correct without landing too far down the 7000-ft runway or running a high risk of a wing scrape while maneuvering. However, in the debriefings at the end of the day almost all of the

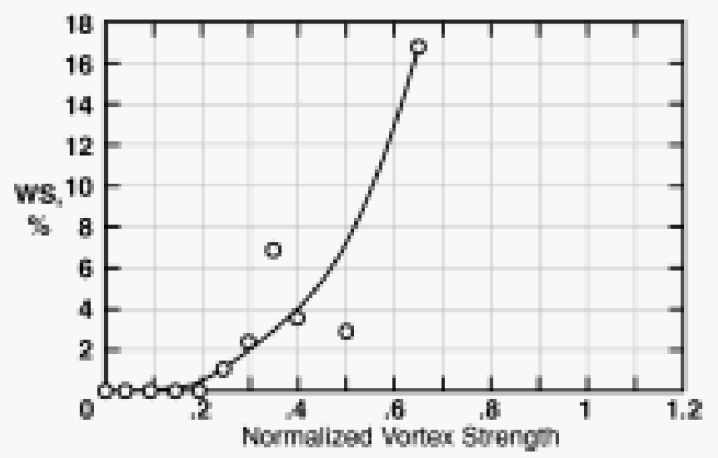

Figure 21. Rate of wing scrapes.

pilots admitted that they had not initiated go-arounds as frequently as they would have in a real airplane for many of the maneuvers. They were aware, at least in their subconscious, that they were flying a simulator and could not damage their equipment or cause injuries. As a result of their failure to execute enough go-arounds, they probably had more wing scrapes than they would have in real airplanes especially if the scrape angle had not been set so low. The most likely scenario for a wing-scrape involved the pilot maneuvering to correct a previous lateral translation offset. As the pilot banked the airplane to stop a lateral drift rate just before touchdown, he encountered a wake that reinforced his deliberate bank angle. The rate of the onset of the wake disturbance was too high for the pilot to prevent the wing from scraping.

Debriefing: The disturbance rating scale, figure 4 , did not contain any references to an "acceptable" or "insignificant" disturbance. However, at the debriefing at the end of the day, the pilots were asked to select two ratings that they personally would consider defined the upper limit of such disturbances. They were asked to define these limits in the context of routine approaches and all levels of experience for other flight crews and not their own personal preferences. The results of this survey are presented in Table III. The opinions varied as much as 3 or 4 rating numbers for different pilots showing the great diversity of opinion on the subject of wake disturbances. However, the average rating estimate for a "nuisance" disturbance was about " 5 " while the average for an "acceptable" disturbance was " 6 ."

Table III.

Estimated ratings for type of disturbances.

\begin{tabular}{|c|c|c|}
\hline & \multicolumn{2}{|c|}{ Rating for disturbance } \\
\hline Pilot & Insignificant & Acceptable \\
\hline 1 & 6 & 7 \\
2 & 5 & 6 \\
3 & 7 & 7 \\
4 & 6 & 7 \\
5 & 5 & 6 \\
6 & 6 & 7 \\
7 & 6 & 6 \\
8 & 4 & 7 \\
9 & 6 & 7 \\
10 & 6 & 7 \\
11 & 3 & 4 \\
12 & 4 & 5 \\
13 & 5 & 6 \\
14 & 4 & 5 \\
\hline Average & 5.2 & 6.2 \\
\hline
\end{tabular}

Sample Application of Results: As stated in the introduction, the main purpose of this study was to provide a means to estimate the vortex strength for a given subjective effect. An example of how to use the preceding results to make such estimates is contained in the following discussion. 
If it is desired to estimate the vortex strength which would produce a "small" disturbance similar to those encountered on a mid afternoon summertime day, a rating of " 6 " would be picked from figure 4 . The next step would be to proceed to figure 9 and decide the desired percentile level of the desired result. For example, a rating of " 6 " at the 50 percentile level would define a normalized vortex strength of 0.2 . Once the percentile level and normalized vortex strength are determined, then the airplane response can also be estimated based on these simulation results. For example, from figure 11, the maximum roll angle for these conditions (vortex strength $=0.2,50$ percentile) would be about 8 degrees. Similar response or touchdown parameters can be estimated from the remaining figures. Of course, the figures can also be used to estimate the subjective and objective responses given a known value of the vortex strength.

The normalized vortex strength can be transformed to an estimate of the absolute vortex strength or circulation level. For example, in the appendix it is estimated that the critical circulation strength of $560 \mathrm{ft} / \mathrm{sec}^{2}$ corresponds to a normalized vortex strength of 0.2 for a 737-200. Similar calculations can be made for other sized airplanes assuming the critical roll acceleration determined in these studies for the B737 is the same for all sized airplanes. These critical circulation strengths can then be compared to the estimated initial circulation strengths of various airplanes as also shown in the appendix.

The results of these calculations are summarized in Table IV. The third column lists the ratio of the critical vortex strength for the follower airplane to the initial vortex strength of the wake from a generator airplane that is identical to the follower airplane. For example, it is estimated that a B737-200 could tolerate a wake from an identical B737-200 after it had decayed to 0.21 times its original strength. For the larger airplanes, the decay does not have to be nearly as much. This trend is due to the fact that, in general, the rolling moment of inertia increases more rapidly than the vortex-induced rolling moment as the airplanes get larger.

The fourth column lists a smaller airplane with an initial vortex strength that the follower could tolerate without any decay. For example, according to these results a B737-200 could only tolerate an initial strength from a Cessna 310 airplane or a smaller airplane. However, the results for the larger airplanes do not appear to be as conservative. For example, the B747400 could tolerate the initial wake strength of a B767200.
It should be remembered that these calculations are based on the assumption that the critical roll acceleration is the same for a large airplane such as the B747 as it is for the smaller B737 simulated in these tests. More studies with large airplanes are needed to determine if this extrapolation is warranted.

Table IV. Estimated Critical Wake Strengths for Various Airplanes for $\ddot{\phi}_{v}=10.6 \mathrm{deg} / \mathrm{sec}^{2}$.

\begin{tabular}{|c|c|c|c|}
\hline $\begin{array}{c}\text { Follower } \\
\text { Airplane }\end{array}$ & $\begin{array}{c}\Gamma_{\text {crit }}, \\
\mathrm{ft}^{2} / \mathrm{sec}\end{array}$ & $\begin{array}{c}\Gamma_{\text {crit }} / \\
\Gamma_{0, \mathrm{f}}\end{array}$ & $\begin{array}{c}\text { Generator Airplane } \\
\text { For } \Gamma_{0, \mathrm{~g}}=\Gamma_{\text {crit }}\end{array}$ \\
\hline $\mathrm{B} 737-200$ & 560 & 0.21 & Cessna 310 \\
\hline $\mathrm{B} 727-200$ & 760 & 0.22 & DHC-6 Twin Otter \\
\hline $\mathrm{B} 757-200$ & 2200 & 0.56 & Foker F-28-4000 \\
\hline $\mathrm{B} 777-200$ & 3600 & 0.71 & $\mathrm{~B} 727-200$ \\
\hline $\mathrm{B} 747-400$ & 4100 & 0.66 & $\mathrm{~B} 767-200 \mathrm{ER} / 300$ \\
\hline
\end{tabular}

Comparison with Previous Studies: The value for the normalized vortex strength of 0.2 is much lower than the 0.5 estimated in a previous study. ${ }^{8}$ Even if an "acceptable" rating was defined as a " 7 " on the rating scale, the 50 percentile normalized vortex strength would only be about 0.3 . Also, if a more conservative 90 percentile rating of " 6 " was desired, the acceptable vortex strength would drop to practically zero. These more conservative answers are understandable given the worst-case methodology used in the present study. If a higher level of acceptable vortex strength is needed for a wake vortex system, additional studies are needed to determine which of the present worst-case assumptions can be safely relaxed. For example, if the repeated disturbance scenario could be shown to be unnecessarily conservative, the resulting strength might be considerably higher.

\section{Concluding Remarks}

A piloted simulation study of simulated lowstrength wake vortex disturbances has been conducted in a research simulator for a B737-100 airplane. A worst-case methodology was used to study rolldominated disturbances on final approach in instrument meteorological conditions. The primary data were the subjective disturbance ratings of the disturbances using a rating scale designed specifically for this study. In addition, several quantitative measures of the approaches were made. The following observations were made:

(1) There were large variations in the disturbance ratings for similar conditions. These variations were due primarily to differences between pilots.

(2) Variations of the simulated lateral control authority appeared to have no significant 
effect on the disturbance ratings. It, therefore, appeared that the value of the roll acceleration due to the vortex was the primary determinant of the subjective disturbance ratings.

(3) Although the approach had three distinctly different segments with different piloting tasks, there were no significant differences in the disturbance ratings for the different segments.

(4) Several charts summarizing the results are presented. These charts can be used to estimate vortex strength for a given subjective rating and percentile level. Estimates of objective airplane/pilot responses can also be made.

(5) Although no specific boundaries were determined between acceptable and unacceptable disturbances, the results of these studies appear to be more conservative than the results of previous studies.

(6) A significant number of pilots rated a zero-wake-strength disturbance as if a disturbance had occurred. They apparently thought the low-level responses to their own control inputs were responses due to a low-strength wake disturbance.

(7) The pilots frequently over controlled and their control inputs were limited by the position and rate limits of the sidearm controller used in this study.

(8) Further studies using simulators with conventional yoke controllers and different sized airplanes are desirable. The most useful study would consist of a few unexpected disturbances for a large number of pilots in equipment they are familiar with. Such a study would require the active involvement of one or more major airline companies.

\section{References}

1. Hinton, D. A., "An Aircraft Vortex Spacing System (AVOSS) for Dynamic Wake Vortex Spacing Criteria," Proceedings of 78th Fluid Dynamics Panel Symposium, Characterization and Modification of Wakes from Lift: Vehicles in Fluids, AGARD, CP-584, Trondheim, Norway, May 1996, pp. 23-1 to 23-12.
2. Stewart, Eric C.,"A Study of the Interaction Between a Wake Vortex and an Encountering Airplane," AIAA Paper No. 93-3642, Monterey, CA, Aug. 1993.

3. Stuever, R. A. and Greene, G. C., "An Analysis of Relative Wake-Vortex Hazards for Typical Transport Aircraft," AIAA Paper No. 94-0810, Reno, NV, Jan. 1994.

4. Thibaudeau, Roger Edward Jr., "On the Dynamics of Transport Aircraft in the Presence of Wake Vortices," M. S. Thesis, George Washington University, September 1997.

5. Brandon, Jay M., Jordan, Frank L. Jr., Buttrill, Catherine W. and Robert A. Stuever, Robert A., "Application of Wind Tunnel Free-Flight Technique for Wake Vortex Encounters," NASA TP-3672, November 1997.

6. Vicroy, D., Brandon, J., Greene, G., Rivers, R., Shah, G., Stewart, E., and Stuever, R., "Characterizing the Hazard of a Wake Vortex Encounter," AIAA Paper No. 97-0055, Reno, NV, Jan. 1997.

7. Sammonds, R.I., Stinnett, G.W. Jr., and Larsen, W. E., "Wake Encounter Hazard Criteria for Two Aircraft Classes," NASA TM X-73113, June 1976.

8. Rossow, V.J., and Tinling, B.E., "Research on Aircraft/Vortex-Wake Interactions to Determine Acceptable Level of Wake Intensity," AIAA Journal of Aircraft. June 1988.

9. Smith, R. Marshall; Chung, Victoria I.; and Martinez, Debbie, "Transport Delays Associated with the NASA Langley Flight Simulation Facility," NASA Technical Memorandum 110150, February 1995.

10. Parrish, Russell, V.; Dieudonne, James E.; Martin, Dennis J., Jr.; and Copeland, James L., "Compensation Based on Linearized Analysis for a Six-Degree-of-Freedom Motion Simulator," NASA TND-7349, November 1973.

11. Stewart, Eric C., "Piloted Simulation Study of Wake Encounters," Proceedings of NASA First Wake Vortex Dynamic Spacing Workshop. NASA/CP-97-206235. November 1997.

12. Defoire, Thomas and Micklos, Richard, "Video Landing Parameter Survey - John F. Kennedy International Airport," DOT/FAA/AR-96/125, July 1997.

13. Rossow, Vernon J., "Inviscid Modeling of Aircraft Trailing Vortices," in Wake Vortex Minimization, NASA SP-409, Feb. 1976. 


\section{Appendix}

The circulation strength, $\Gamma$, of a vortex corresponding to a given non-dimensional normalized vortex strength can be easily estimated for simple geometries. One of the simplest geometries which is still conservative is a single vortex centered on the wing of the encountering airplane. Using the Burnham model of a vortex, where $V_{\theta}$ is the tangential vortex velocity at a radius $r$ from the center of the vortex and $r_{c}$ is the radius of the vortex core,

$$
\mathrm{V}_{\theta}=(\Gamma / 2 \pi)\left\{\mathrm{r} /\left(\mathrm{r}^{2}+\mathrm{r}_{\mathrm{c}}^{2}\right)\right\}
$$

a closed-form solution for the rolling moment, $\mathrm{M}_{\mathrm{x}}$, can be obtained as a function of the wing lift curve slope, $\mathrm{cl}_{\alpha}$; area, $\mathrm{S}$; span, $\mathrm{b}$; and taper ratio, $\lambda$; true airspeed, $\mathrm{V}_{\infty}$; and air density, $\rho$.

$$
\begin{gathered}
\mathrm{M}_{\mathrm{x}}=\left\{\Gamma \rho \mathrm{V}_{\infty} \mathrm{c}_{\alpha} / 4 \pi\right\}\{2 \mathrm{~S} / \mathrm{b}(\lambda+1)\} \\
\left\{[2(\lambda-1) / \mathrm{b}]\left[(\mathrm{b} / 2)^{2}-\mathrm{r}_{\mathrm{c}}{ }^{2} \log \left|(\mathrm{b} / 2)^{2}+\mathrm{r}_{\mathrm{c}}{ }^{2}\right|+\mathrm{r}_{\mathrm{c}}{ }^{2} \log \left|\mathrm{r}_{\mathrm{c}}{ }^{2}\right|\right]+\left[\mathrm{b}-2 \mathrm{r}_{\mathrm{c}} \tan ^{-1}\left(\mathrm{~b} / 2 \mathrm{r}_{\mathrm{c}}\right)\right]\right\}
\end{gathered}
$$

or

$$
\mathrm{M}_{\mathrm{x}}=\Gamma \cdot \mathrm{f}\left(\mathrm{b}, \mathrm{s}, \mathrm{c}_{\alpha}, \lambda, \rho, \mathrm{V}_{\infty}, \mathrm{r}_{\mathrm{c}}\right)
$$

This solution shows that the rolling moment is proportional to the circulation strength of the vortex. With knowledge of the moment of inertia of the airplane, the rolling acceleration can be easily calculated for any circulation strength.

$$
\ddot{\phi}_{v}=\left(\mathrm{M}_{\mathrm{X}} / \mathrm{I}_{\mathrm{XX}}\right) \cdot 57.3
$$

Finally, from the definition of the normalized vortex strength and the maximum acceleration due to full deflection of the lateral controls in Table II $\left(53 \mathrm{deg} / \mathrm{sec}^{2}\right)$, the circulation strength can be calculated as a function of normalized vortex strength.

$$
\ddot{\phi_{v}}=(\text { Normalized Vortex Strength })\left(53 \mathrm{deg} / \mathrm{sec}^{2}\right)=\left(\mathrm{M}_{\mathrm{x}} / \mathrm{I}_{\mathrm{XX}}\right) \cdot 57.3
$$

These formulas can be used to estimate the critical maximum circulation strength, $\Gamma_{\text {crit }}$, that can be encountered by any airplane for a given value of normalized vortex strength (in these calculations, 0.2 ). That is, assuming the fundamental metric for disturbances is the roll acceleration and the critical value $(0.2$ $\mathrm{x} 53=10.6 \mathrm{deg} / \mathrm{sec}^{2}$ ) is independent of the airplane and its size, a value for $\Gamma_{\text {crit }}$ was calculated for the following airplanes with the assumed characteristics in the table and with $\mathrm{r}_{\mathrm{c}}=2 \mathrm{ft}$ and $\rho=.002378$. The values for $\mathrm{c}_{\alpha}$, which are crucial to these calculations, were estimated using the geometric characteristics of the wing. ${ }^{13}$ 
Table IA. Assumed follower airplane characteristics and estimated critical circulation strengths.

\begin{tabular}{|c|c|c|c|c|c|c|c|}
\hline $\begin{array}{c}\text { Follower } \\
\text { Airplane }\end{array}$ & $\begin{array}{c}\mathrm{V}_{\infty} \\
\mathrm{ft} / \mathrm{sec}\end{array}$ & $\begin{array}{c}\mathrm{c}_{1_{\alpha},} \\
\text { per radian }\end{array}$ & $\begin{array}{c}\mathrm{S}, \\
\mathrm{ft}^{2}\end{array}$ & $\begin{array}{c}\mathrm{b}, \\
\mathrm{ft}\end{array}$ & $\begin{array}{c}\lambda, \\
\text { non- } \\
\text { dimensional }\end{array}$ & $\begin{array}{c}\mathrm{I}_{\mathrm{Xx}} \\
\text { slug-ft }\end{array}$ & $\begin{array}{c}\Gamma_{\text {crit }}, \\
\mathrm{ft}^{2} / \mathrm{sec}\end{array}$ \\
\hline $\mathrm{B} 737-200$ & 218 & 3.46 & 1098 & 93 & .34 & 432,000 & 560 \\
\hline $\mathrm{B} 727-200$ & 225 & 3.09 & 1700 & 108 & .3 & 847,000 & 760 \\
\hline B757-200 & 231 & 3.39 & 1951 & 124.5 & .23 & $3,182,000$ & 2200 \\
\hline B777-200 & 233 & 3.36 & 4605 & 199.9 & .2 & $12,714,000$ & 3600 \\
\hline B747-400 & 258 & 2.98 & 6360 & 211 & .25 & $19,600,000$ & 4100 \\
\hline
\end{tabular}

These critical circulation strengths can be put into practical terms by expressing them as either (1) ratios to the initial strength of the wake of a generator airplane of the same type as the follower or (2) equivalencies to another (smaller) airplane with approximately the same initial circulation strength. This can be accomplished by first estimating the initial circulation strength of a given airplane using the following formula

$$
\Gamma_{0}=(4 / \pi)\left\{(\text { Weight }) /\left(\rho V_{\infty} b\right\}\right.
$$

The estimated initial vortex strengths for 9 airplanes needed for these practical expressions are given in the following table

Table IIA. Estimated Initial Wake Strengths for Several Typical Airplanes.

\begin{tabular}{|c|c|c|c|c|}
\hline Generator Airplane & $\begin{array}{c}\text { Weight, } \\
\mathrm{lb}\end{array}$ & $\begin{array}{c}\mathrm{b}, \\
\mathrm{ft}\end{array}$ & $\begin{array}{c}\mathrm{V}_{\infty}, \\
\mathrm{ft} / \mathrm{sec}\end{array}$ & $\begin{array}{c}\Gamma_{0}, \\
\mathrm{ft}^{2} / \mathrm{sec}\end{array}$ \\
\hline Cessna 310 & 5400 & 36.9 & 147 & 533 \\
\hline DHC-6 Twin Otter & 12300 & 65 & 127 & 800 \\
\hline Foker F-28-4000 & 69500 & 82.25 & 205 & 2200 \\
\hline B737-200 & 103,000 & 93 & 218 & 2720 \\
\hline B727-200 & 154,500 & 108 & 225 & 3400 \\
\hline B757-200 & 210,000 & 124.5 & 231 & 3910 \\
\hline B767-200ER/300 & 285000 & 156.1 & 235 & 4160 \\
\hline B777-200 & 445,000 & 199.9 & 233 & 5120 \\
\hline B747-400 & 630,000 & 211 & 258 & 6200 \\
\hline
\end{tabular}

